\title{
Bladder mucosa-associated lymphoid tissue lymphoma progressed from chronic cystitis along with a comparative genetic analysis during long-term follow-up: a case report
}

\author{
Naoya Ishibashi ${ }^{1}$, Yoko Nakanishi ${ }^{2}$, Haruna Nishimaki ${ }^{2}$, Toshiya Maebayashi ${ }^{1}$, Shinobu Masuda ${ }^{2}$, \\ Masahiro Okada ${ }^{1}$ \\ ${ }^{1}$ Department of Radiology, Nihon University School of Medicine, Itabashi, Tokyo, Japan; ${ }^{2}$ Department of Oncologic Pathology, Nihon University \\ School of Medicine, Itabashi, Tokyo, Japan \\ Correspondence to: Naoya Ishibashi. Department of Radiology, Nihon University School of Medicine, 30-1 Oyaguchi Kami-cho Itabashi-ku, Tokyo \\ 173-8610, Japan. Email: ishibashi.naoya@nihon-u.ac.jp.
}

\begin{abstract}
The pathogenesis of bladder marginal zone/mucosa-associated lymphoid tissue (MALT) lymphoma, which is the most common type of primary bladder lymphoma, has not been clarified. There are no reports that described histological and molecular time course of MALT lymphoma occurring in the bladder and the importance of the score on the Pelvic Pain and Urgency/Frequency (PUF) patient symptom scale during and after radiation therapy (RT). We present a case of MALT lymphoma with long-term comparative genetic analysis. A 77-year-old Japanese woman with hematuria and severe perineal pain was found to have a tumor-like lesion in the bladder trigone. She was diagnosed with cystitis based on the results of pathological examination and immunostaining after transurethral resection of the lesion. The second transurethral resection procedure was performed approximately 4 years after the first procedure because of recurrence of the hematuria and enlargement of a lesion in the left bladder wall. Postoperative pathologic examination confirmed a diagnosis of MALT lymphoma. Genetic analysis of immunoglobulin heavy chain $(I G H)$ gene rearrangements showed more clonal progression from the first biopsy to the second. The patient then underwent RT, during which her perineal pain was exacerbated by radiation cystitis but finally decreased to a level less severe than that before treatment. The PUF patient symptom scale was useful to monitor her pain throughout the clinical course. No recurrence was detected more than 2 years after completion of RT.
\end{abstract}

Keywords: Bladder mucosa-associated lymphoid tissue lymphoma; pelvic pain and urgency/frequency symptom scale; radiation therapy; case report

Submitted Jul 08, 2021. Accepted for publication Sep 12, 2021.

doi: 10.21037/tau-21-602

View this article at: https://dx.doi.org/10.21037/tau-21-602

\section{Introduction}

Primary bladder lymphoma is a very rare disease, accounting for less than $1 \%$ of all primary bladder tumors and $0.15 \%$ to $0.20 \%$ of extranodal lymphomas (1-3). The most common subtype of primary bladder lymphoma is low-grade extranodal marginal zone/mucosa-associated lymphoid tissue (MALT) lymphoma, which accounts for approximately half of all primary bladder lymphomas $(4,5)$. Cystitis due to chronic inflammation is considered a cause of bladder lymphoma because lymphoid tissue is typically absent in the bladder (4-6). We describe a patient with a bladder tumor and severe intractable perineal pain, which progressed from cystitis to MALT lymphoma. The score on the Pelvic Pain and Urgency/Frequency (PUF) patient symptom scale (7) transiently worsened during radiation therapy (RT) but dramatically improved after successful treatment. In previous reports of bladder lymphoma, the histological and molecular time course of MALT lymphoma from cystitis and changes in the PUF patient symptom scale have not been examined in the same patient. We present the following case in 

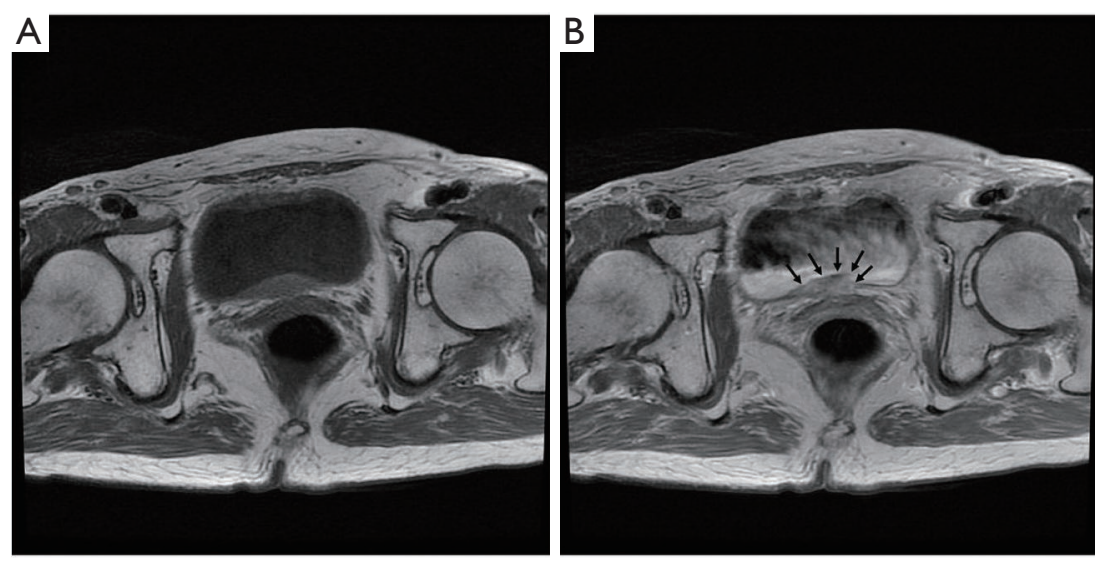

Figure 1 Magnetic resonance imaging before the first transurethral resection of the bladder tumor (A). A gadolinium enhanced T1weighted image revealed a strongly enhanced elevated lesion in the bladder trigone (arrows) (B).

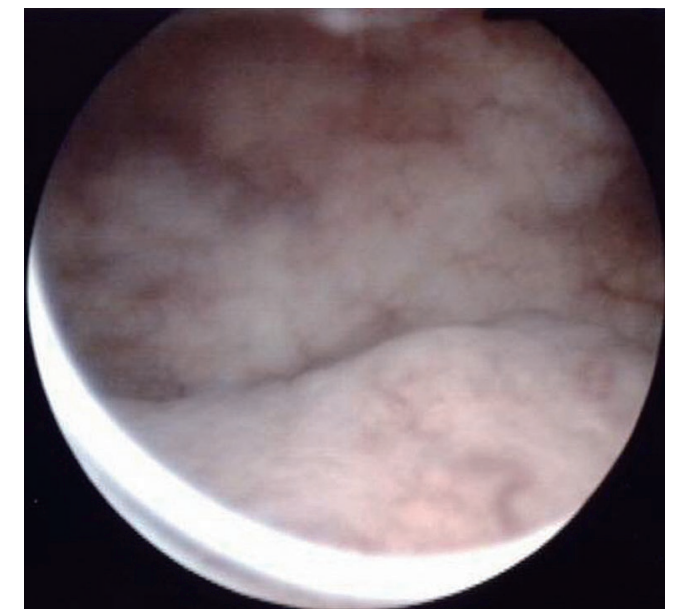

Figure 2 Cystoscopy image before the first transurethral resection of the bladder tumor. A submucosal tumor-like lesion was seen.

accordance with the CARE reporting checklist (available at https://dx.doi.org/10.21037/tau-21-602).

\section{Case presentation}

A 77-year-old Japanese woman with symptoms of hematuria and severe perineal pain visited our hospital. The patient had experienced pollakiuria for seven years. Cystoscopy had never been performed. Urine sediment showed white blood cells, urine culture showed escherichia coli but urine cytology showed no malignant cells at this time. Magnetic resonance imaging performed at our hospital revealed a strongly enhanced elevated lesion in the bladder trigone (Figure 1); cystoscopy suggested that it was a submucosal tumor-like lesion (Figure 2). Transurethral resection of the bladder tumor (TUR-BT) was performed. Pathological examination with hematoxylin-eosin ( $\mathrm{H} \& \mathrm{E})$ staining showed no epithelial tumor. Although marked infiltration of lymphocytes was observed in the mucosa, they were not atypical lymphocytes (Figure 3). Immunostaining revealed CD20 positive $\mathrm{B}$ cells and $\mathrm{CD} 3$ positive $\mathrm{T}$ cells intermingled without monoclonality. Accordingly, the patient was diagnosed with cystitis. She was treated with various antibacterial drugs and antiallergic drugs. After the first TUR-BT, urine cytology showed just mildly atypical urothelial cells with a few neutrophils (Figure 4). Her symptoms improved and worsened repeatedly during follow-up. However, her hematuria relapsed, and magnetic resonance imaging showed enlargement of an irregular elevated lesion in the left bladder wall (Figure 5) and urine cytology showed that small to medium sized atypical lymphocytes with irregular nuclei and sparse chromatins in a sporadic or mass were observed. Approximately 4 years after the first TUR-BT, the second TUR-BT was performed. The cystoscopy suggested that it was non-papillary nodular tumor (Figure 6). Pathological examination with $\mathrm{H} \& \mathrm{E}$ staining showed marked infiltration of lymphocytes in the mucosa. In contrast to the previous findings, lymphocytes showed increased atypia and diffuse and stronger expression for CD20. There were fewer CD3 positive cells than before. Atypical lymphocytes were B-cell lymphoma (Bcl)-2 positive and bcl-6 negative; thus, MALT lymphoma was diagnosed. Polymerase chain reaction (PCR) and fluorescence in situ hybridization (FISH) were 

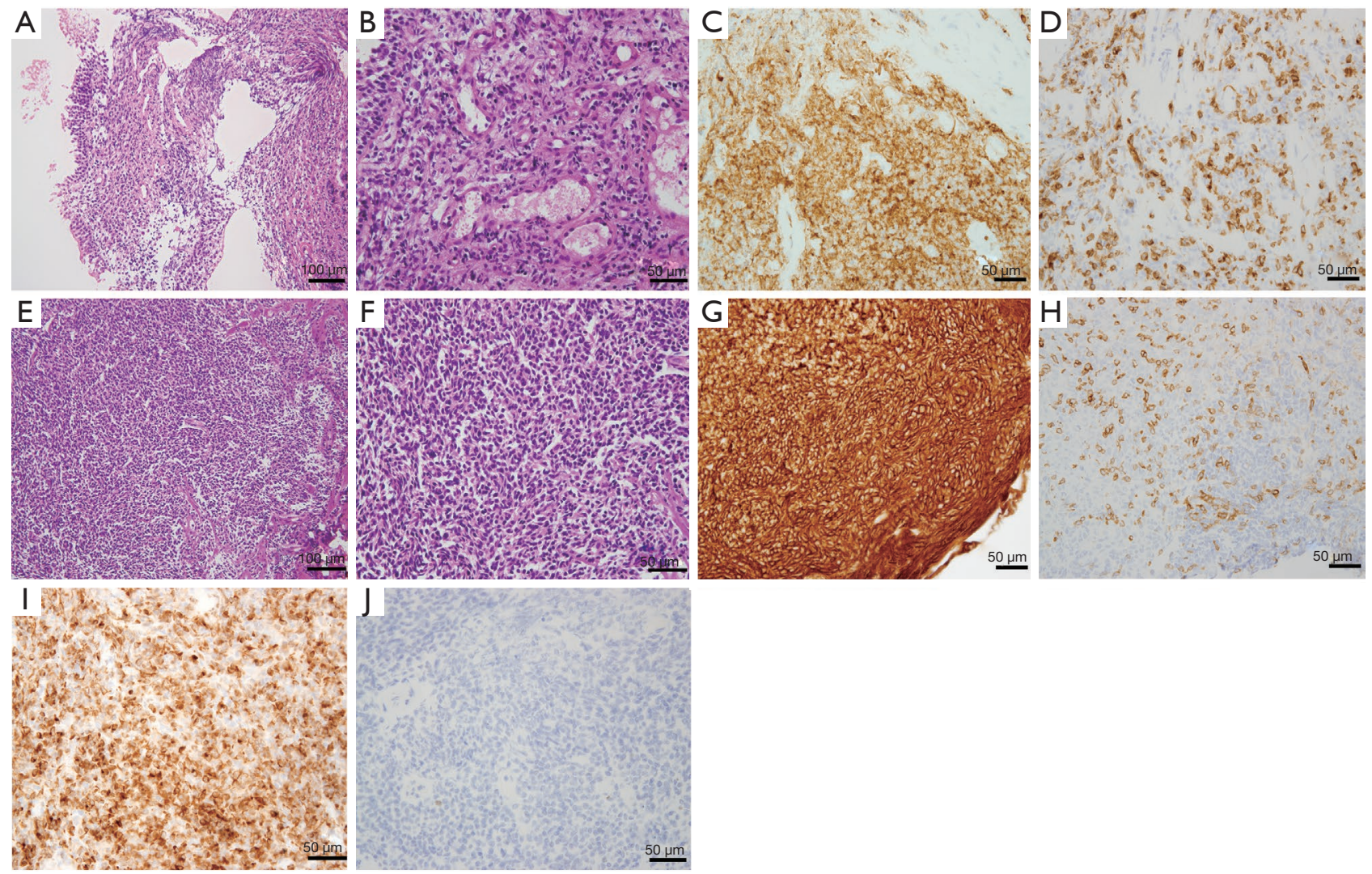

Figure 3 Histological and immunophenotypic features of two biopsy samples over 4 years. H\&E staining of the first sample (A-D) showed lymphocytes in the mucosa, but not atypical (A, 40× magnification; B, 100× magnification). (C) CD20 positive cells and (D) CD3 positive cells are intermingled without monoclonality, thus, the first sample are diagnosed as cystitis. H\&E staining of the second sample (E-H) showed lymphocytes with increased atypia and diffuse staining pattern than before (E, 40× magnification; F, 100× magnification). (G) CD20 expression are stronger and diffuse than before. $(\mathrm{H}) \mathrm{CD} 3$ positive cells are fewer than before. (I) Bcl-2 was positive and (J) Bcl-6 was negative in the second sample. Thus, the second sample was diagnosed as MALT lymphoma.

performed to compare the genetic characteristics of the two biopsy samples obtained before and after 4-year followup period. The BIOMED-2 primer system was used to analyze immunoglobulin gene clonal rearrangements using multiplex PCR, according to the BIOMED-2 guidelines $(8,9)$. Immunoglobulin heavy chain $(I G H)$ gene rearrangements in the first and the second sample were compared with a normal tonsil sample. By electrophoreses, a single band was detected only in the second sample. These results showed that the tumor cell growth in the second sample progressed with more clonal $I G H$ gene rearrangement than in the first sample (Figure 7). A translocation of $\mathrm{t}(14 ; 18)(\mathrm{q} 32 ; \mathrm{q} 21)$, involving IGH at $14 \mathrm{q} 32$ and MALT1 at $18 \mathrm{q} 21$, is a recurrent abnormality detected in MALT lymphoma (10). Therefore, we investigated the fusion gene by FISH techniques using Cytocell aquarius $\mathrm{t}(14 ; 18)$ (32.33:21.31-21.32)/IGH-MALT1 dual fusion probe (Cytocell Ltd., Cambridge, UK). However, IGHMALT1 fusion signals were not detected in the first nor second sample (data not shown). No infiltration in any other organs was detected using either fluorodeoxyglucosepositron emission tomography or computed tomography, and the MALT lymphoma was staged as Ann Arbor stage $I_{E}(11)$. The patient elected to undergo RT. Because her severe perineal pain was expected to be transiently exacerbated by radiation cystitis, we provided an explanation to the patient and obtained consent for RT. The patient independently completed the PUF patient symptom scale from before starting to after completing the treatment. In the PUF scale, the patient answers questions regarding 


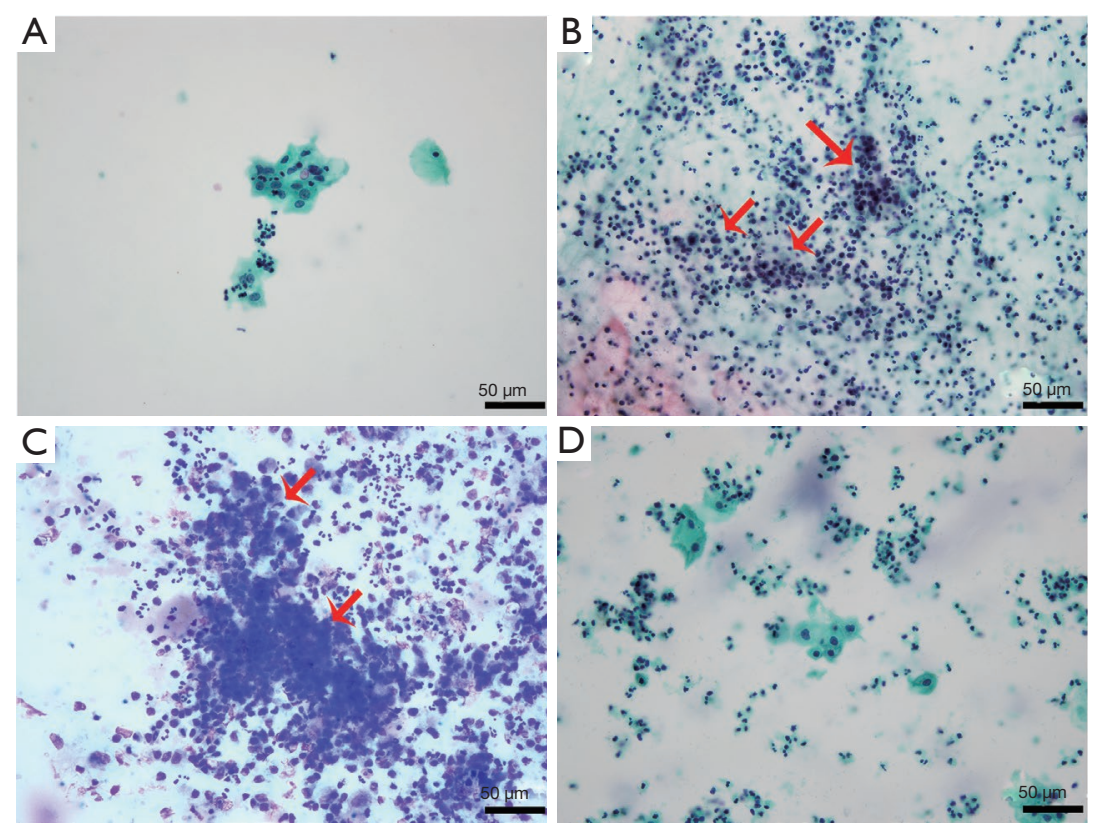

Figure 4 Urine cytological features of two biopsy samples over 5 years. (A) After the first transurethral resection of the bladder tumor (TUR-BT), just mildly atypical urothelial cells with a few neutrophils were seen. (B,C) Before the second TUR-BT, small to medium sized atypical lymphocytes with irregular nuclei and sparse chromatins in a sporadic or mass were observed (red arrows). (D) After completing RT, reactive inflammatory changes with a large number of neutrophils and mildly atypical urothelial cells were observed. Staining method: papanicolaou stain (A,B,D) and giemsa stain (C).
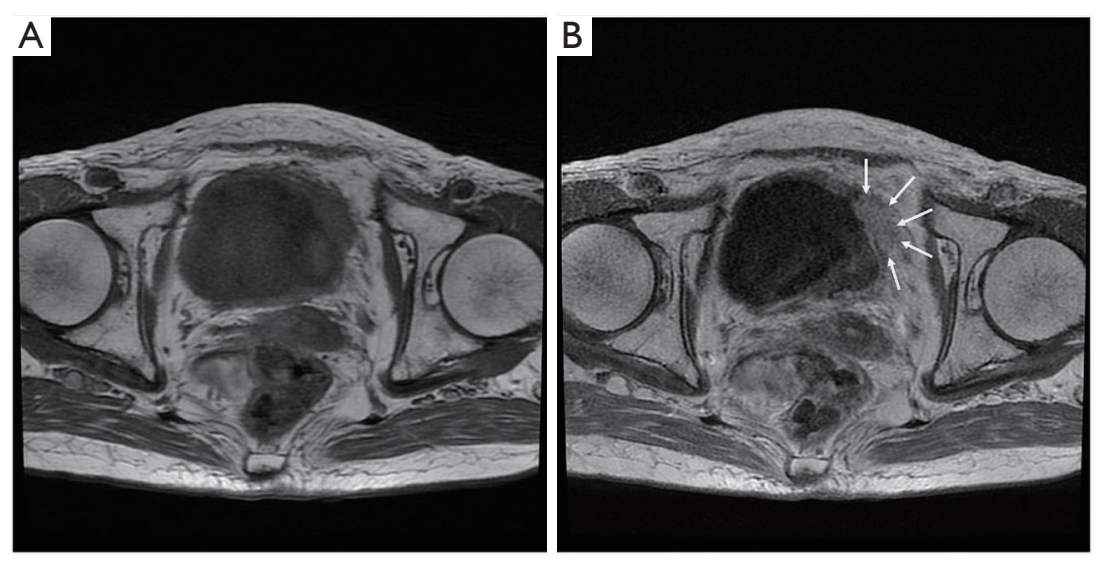

Figure 5 Magnetic resonance imaging before the second transurethral resection of the bladder tumor (A). A gadolinium enhanced T1weighted image revealed an irregular elevated lesion in the left bladder wall (white arrows) (B).

how he or she feels with regard to pelvic pain and urgency/ frequency. Each score ranges from 0 points (best) to 3 or 4 points (worst), and the total score is the sum of the symptom score and bother score (7). The whole bladder was determined as the clinical target volume, and the patient underwent three-dimensional conformal RT with 10-MV photon beams using the box technique at a dose of $1.8 \mathrm{~Gy} /$ fraction (30.6 Gy in total). The PUF score was 15 before starting RT, increased to 18 on the 8 th day after starting RT, and continued to increase thereafter, reaching a peak of 29 on the 29th day after starting RT. The score dramatically decreased thereafter and reached 8 on the 447 th day after 


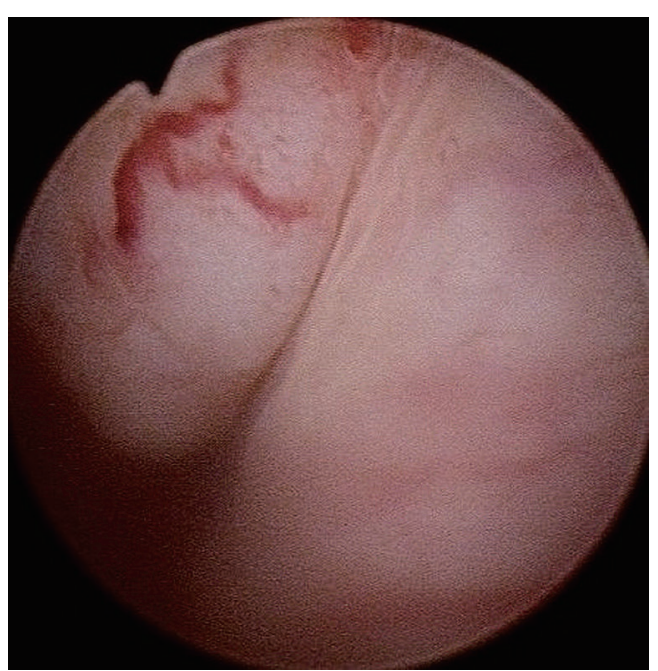

Figure 6 Cystoscopy image before the second transurethral resection of the bladder tumor. A non-papillary nodular tumor was seen.

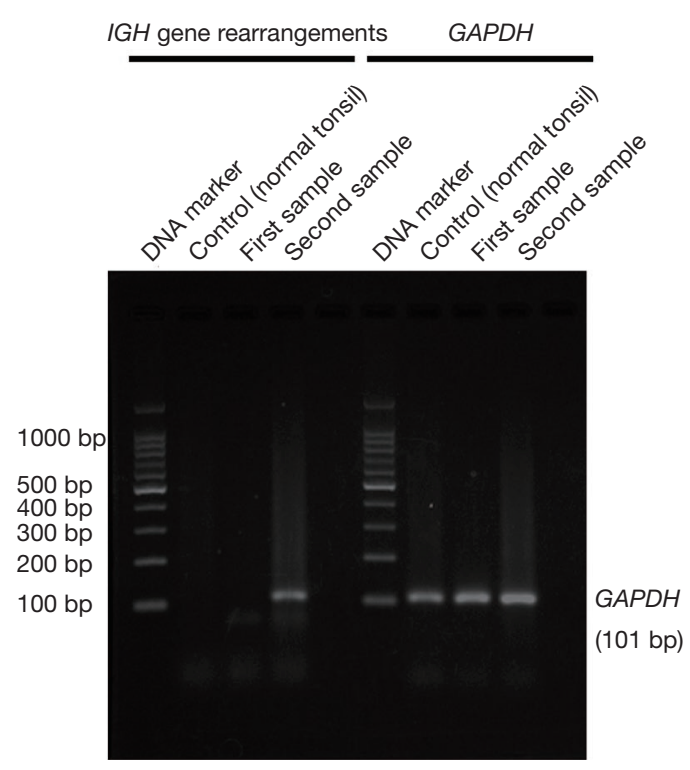

Figure 7 PCR analysis to investigate immunoglobulin heavy chain $(I G H)$ gene rearrangements in the first and the second sample after over 4 years. Normal tonsil was used as control sample. IGH gene rearrangements and internal control glyceraldehyde 3-phosphate dehydrogenase (GAPDH) were shown.

completing RT, remaining lower than the score before starting RT (Figure 8). Cystoscopy showed disappearance of the lesion on the 517th day after completing RT (Figure 9). Urine sediment showed white blood cells and urine culture showed escherichia coli during follow-up. Urine cytology showed that reactive inflammatory changes with a large number of neutrophils and mildly atypical urothelial cells were observed (Figure 4D). No recurrence was observed at the time of this writing (1098th day after completing RT).

All procedures performed in studies involving human participants were in accordance with the ethical standards of the institutional and/or national research committee(s) and with the Helsinki Declaration (as revised in 2013). Written informed consent was obtained from the patient for publication of this case report and accompanying images. A copy of the written consent is available for review by the editorial office of this journal.

\section{Discussion}

Bladder MALT lymphoma may originate from underlying prolonged chronic inflammation of the bladder (4-6), though precise mechanisms have not been clarified, unlike Helicobacter pylori infection in gastric MALT $(12,13)$. Lymphoepithelial lesion (LEL) has been reported one of characteristic findings of MALT lymphoma in general, and can exist in areas of cystitis glandularis of the bladder without lymphoid tissue (14-16). LEL is rare in patients with bladder MALT lymphoma, and its identification is not essential for diagnosis. The presence of LEL was not clear in our patient. Many cases of bladder MALT lymphoma unrelated to cystitis have also been reported, so whether these conditions are truly related remains unclear $(6,17,18)$. In our patient, we confirmed that bladder MALT lymphoma developed from underlying cystitis after 4 years, and we are the first to report the results of comparative genetic analyses over time in such a case.

The location of initial cystitis differed from that of MALT lymphoma. We have inferred that proliferative lymphocytes can be seen in the whole bladder with cystitis and MALT lymphoma may occur metachronously in multiple lesions under the same condition like bladder cancer in situ. Few previous reports have focused on the genetic analysis of bladder lymphoma; we identified only six cases in three reports within the scope of our investigation. Among these cases, a monoclonal gene rearrangement of IGH was identified by PCR in five patients $(16,19,20)$. FISH was performed in only one patient, and gene translocation of IGH-MALT1 was confirmed (20). There have been no reports confirming the clonal gene rearrangement of IGH in MALT lymphoma, originating from underlying cystitis with clonal evolution, in the same patient. 


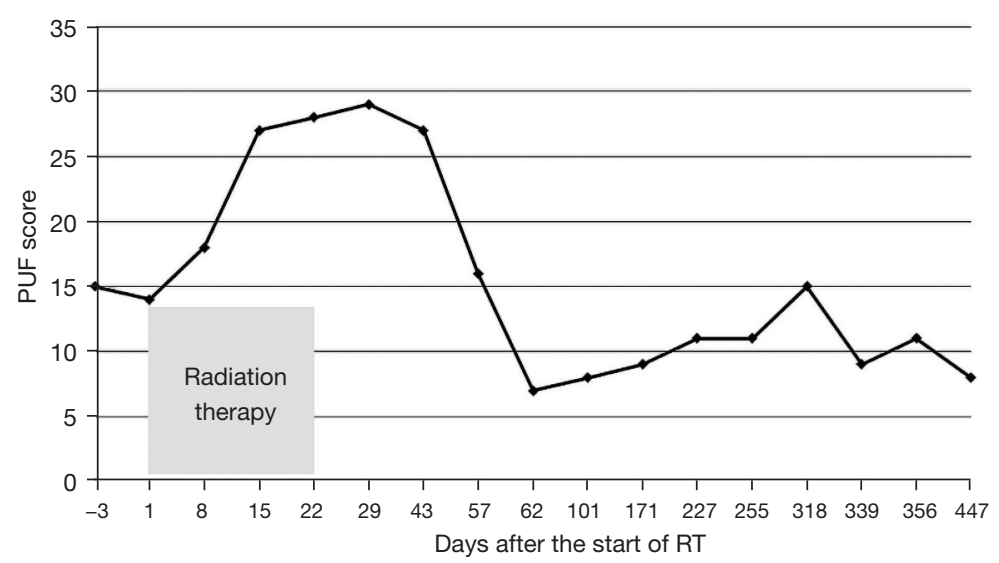

Figure 8 Scores on the Pelvic Pain and Urgency/Frequency (PUF) patient symptom scale from before starting to after completing RT. The score was 15 before starting RT, increased to 18 on the 8th day after starting RT, and continued to increase thereafter, reaching a peak of 29. The score then dramatically decreased and remained lower than the score before starting RT.

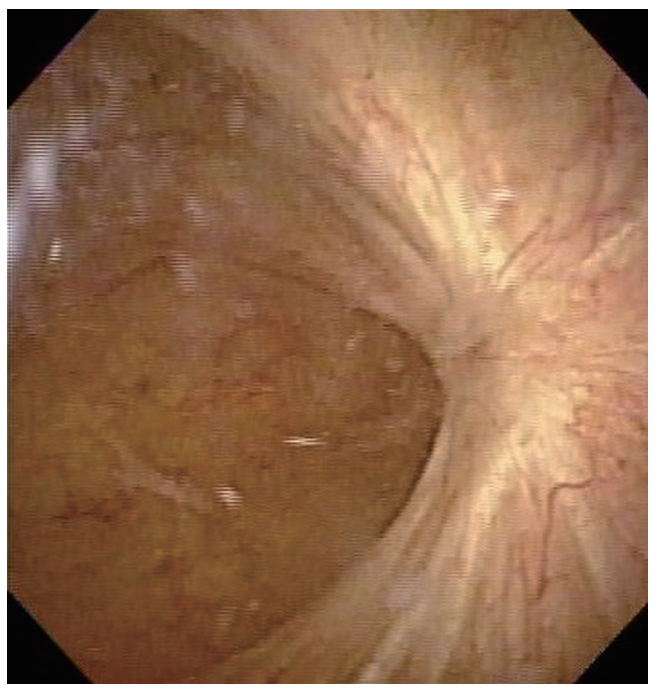

Figure 9 Cystoscopy image on the 517 th day after completing radiation therapy. The tumor lesion disappeared only with bladder wall twitch.

The typical symptoms of bladder lymphoma include urinary frequency, hematuria and suprapubic pain, which are similar to symptoms of cystitis $(15,21)$. The PUF patient symptom scale emphasizes pelvic pain, which is highly correlated with an intravesical potassium sensitivity test that detects bladder epithelial abnormalities (7). Our patient had severe perineal pain, and the scale was useful. The severe perineal pain was transiently aggravated by radiation cystitis as expected, and the PUF score peaked immediately after completing the treatment; however, it then dramatically decreased to below the pretreatment score. Although the standard treatment for bladder lymphoma has not been established, RT after TUR-BT is often performed because it is expected to result in a favorable prognosis $(6,16,21)$. Severe perineal pain is often transiently aggravated by radiation cystitis. However, because the pain will gradually improve, clinicians should not hesitate to use RT.

\section{Acknowledgments}

We thank Angela Morben, DVM, ELS, from Edanz Group (https://en-author-services.edanzgroup.com/ac), for editing a draft of this manuscript.

Funding: None.

\section{Footnote}

Reporting Checklist: The authors have completed the CARE reporting checklist. Available at https://dx.doi. org/10.21037/tau-21-602

Conflicts of Interest: All authors have completed the ICMJE uniform disclosure form (available at https://dx.doi. org/10.21037/tau-21-602). The authors have no conflicts of interest to declare.

Ethical Statement: The authors are accountable for all aspects of the work in ensuring that questions related to the accuracy or integrity of any part of the work are 
appropriately investigated and resolved. All procedures performed in studies involving human participants were in accordance with the ethical standards of the institutional and/or national research committee(s) and with the Helsinki Declaration (as revised in 2013). Written informed consent was obtained from the patient for publication of this case report and accompanying images. A copy of the written consent is available for review by the editorial office of this journal.

Open Access Statement: This is an Open Access article distributed in accordance with the Creative Commons Attribution-NonCommercial-NoDerivs 4.0 International License (CC BY-NC-ND 4.0), which permits the noncommercial replication and distribution of the article with the strict proviso that no changes or edits are made and the original work is properly cited (including links to both the formal publication through the relevant DOI and the license). See: https://creativecommons.org/licenses/by-nc-nd/4.0/.

\section{References}

1. Curry NS, Chung CJ, Potts W, et al. Isolated lymphoma of genitourinary tract and adrenals. Urology 1993;41:494-8.

2. Arda K, Ozdemir G, Güneş Z, et al. Primary malignant lymphoma of the bladder. A case report and review of the literature. Int Urol Nephrol 1997;29:319-22.

3. Wazait HD, Chahal R, Sundurum SK, et al. MALTtype primary lymphoma of the urinary bladder: clinicopathological study of 2 cases and review of the literature. Urol Int 2001;66:220-4.

4. Hughes M, Morrison A, Jackson R. Primary bladder lymphoma: management and outcome of 12 patients with a review of the literature. Leuk Lymphoma 2005;46:873-7.

5. Sellman DP, Simpson WG, Klaassen Z, et al. Characterization and outcomes of local treatment for primary bladder lymphoma: A population-based cohort analysis. Urol Ann 2018;10:249-53.

6. Ohsawa M, Aozasa K, Horiuchi K, et al. Malignant lymphoma of bladder. Report of three cases and review of the literature. Cancer 1993;72:1969-74.

7. Parsons CL, Dell J, Stanford EJ, et al. Increased prevalence of interstitial cystitis: previously unrecognized urologic and gynecologic cases identified using a new symptom questionnaire and intravesical potassium sensitivity. Urology 2002;60:573-8.
8. van Dongen JJ, Langerak AW, Brüggemann $M$, et al. Design and standardization of PCR primers and protocols for detection of clonal immunoglobulin and T-cell receptor gene recombinations in suspect lymphoproliferations: report of the BIOMED-2 Concerted Action BMH4CT98-3936. Leukemia 2003;17:2257-317.

9. $\mathrm{Lu} \mathrm{C}, \mathrm{He} \mathrm{Q}, \mathrm{Zhu} \mathrm{W}$, et al. The value of detecting immunoglobulin gene rearrangements in the diagnosis of B-cell lymphoma. Oncotarget 2017;8:77009-19.

10. Murga Penas EM, Callet-Bauchu E, Ye H, et al. The $\mathrm{t}(14 ; 18)(\mathrm{q} 32 ; \mathrm{q} 21) / \mathrm{IGH}-\mathrm{MALT} 1$ translocation in MALT lymphomas contains templated nucleotide insertions and a major breakpoint region similar to follicular and mantle cell lymphoma. Blood 2010;115:2214-9.

11. Carbone PP, Kaplan HS, Musshoff K, et al. Report of the Committee on Hodgkin's Disease Staging Classification. Cancer Res 1971;31:1860-1.

12. Vallina E, Fresno F, Alonso JL, et al. Incidence of primary gastric lymphoma and $\mathrm{H}$. pylori infection in the central zone of Asturias. An Med Interna 1999;16:175-7.

13. Guindi M. Role of Helicobacter pylori in the pathogenesis of gastric carcinoma and progression of lymphoid nodules to lymphoma. Can J Gastroenterol 1999;13:224-7.

14. Pawade J, Banerjee SS, Harris M, et al. Lymphomas of mucosa-associated lymphoid tissue arising in the urinary bladder. Histopathology 1993;23:147-51.

15. Kempton CL, Kurtin PJ, Inwards DJ, et al. Malignant lymphoma of the bladder: evidence from 36 cases that low-grade lymphoma of the MALT-type is the most common primary bladder lymphoma. Am J Surg Pathol 1997;21:1324-33.

16. Al-Maghrabi J, Kamel-Reid S, Jewett M, et al. Primary low-grade B-cell lymphoma of mucosa-associated lymphoid tissue type arising in the urinary bladder: report of 4 cases with molecular genetic analysis. Arch Pathol Lab Med 2001;125:332-6.

17. Bates AW, Norton AJ, Baithun SI. Malignant lymphoma of the urinary bladder: a clinicopathological study of 11 cases. J Clin Pathol 2000;53:458-61.

18. Simpson RH, Bridger JE, Anthony PP, et al. Malignant lymphoma of the lower urinary tract. A clinicopathological study with review of the literature. $\mathrm{Br}$ J Urol 1990;65:254-60.

19. Ueno Y, Sakai H, Tsuruta T, et al. Mucosa-associated lymphoma of the bladder with relapse in the stomach after successful local treatment. Hinyokika Kiyo 2007;53:575-9.

20. Lucioni M, Nicola M, Riboni R, et al. Antibiotic therapy-induced remission of bladder mucosa-associated 
lymphoid tissue (MALT) lymphoma carrying t $(11 ; 18)$ (q21;q21) apoptosis inhibitor 2-MALT1. J Clin Oncol 2013;31:e304-6.

Cite this article as: Ishibashi N, Nakanishi Y, Nishimaki H, Maebayashi T, Masuda S, Okada M. Bladder mucosa-associated lymphoid tissue lymphoma progressed from chronic cystitis along with a comparative genetic analysis during long-term follow-up: a case report. Transl Androl Urol 2021;10(10): 3899-3906. doi: 10.21037/tau-21-602
21. Vempati P, Knoll MA, Alqatari M, et al. MALT Lymphoma of the Bladder: A Case Report and Review of the Literature. Case Rep Hematol 2015;2015:934374. 